\title{
TIME-PERIODIC SOLUTIONS \\ FOR A SCALAR CONSERVATION LAW
}

\author{
Shigeharu Takeno \\ Department of Information and Electronics Engineering, \\ Niigata Institute of Technology \\ Kashiwazaki, Niigata, 945-1195, JAPAN \\ (E-mail: shige@iee.niit.ac.jp)
}

\begin{abstract}
We consider the existence of time periodic solutions for a nonlinear hyperbolic scalar conservation law with a time periodic outer force. The uniform asymptotic behavior of the Lax-Friedrichs difference approximation gives fixed points of the Poincare map and the convergence of the approximate periodic solution made from such fixed points is proved by the compensated compactness theory.
\end{abstract}

Keywords: time periodic solution, conservation law, periodic outer force, compensated compactness, Lax-Friedrichs difference scheme, Brouwer's fixed point theorem. 


\section{Introduction}

In this paper we study a scalar conservation law with an outer force term

$$
u_{t}+f(u)_{x}=g(t, x)
$$

where the function $f(u)$ is smooth and convex. The global existence of weak solutions of the Cauchy problem (1) for any large initial data was proved in [11]. If initial data $u_{0}(x)$ and an outer force $g(t, x)$ are $x$-periodic functions then the solution $u(t, x)$ may be periodic and may be regarded as a solution of the initial-boundary value problem (1) and

$$
\begin{cases}u(0, x)=u_{0}(x) & (0<x<1), \\ u(t, 0)=u(t, 1) & (t>0) .\end{cases}
$$

We consider the following problem:

If $g(t, x)$ is a time-periodic function with period $T$, does a timeperiodic solution exist with the same period, assuming the necessary condition

$$
\int_{0}^{T} d t \int_{0}^{1} g(t, x) d x=0
$$

for the outer force term $g(t, x)$ ?

For the existence of a time periodic solution, we need the decay of the solution to the homogeneous equation

$$
u_{t}+f(u)_{x}=0
$$

with data (2). The decay to the mean value

$$
\bar{u}=\int_{0}^{1} u(t, x) d x=\int_{0}^{1} u_{0}(x) d x
$$

was obtained under some regularity assumption for the solution at a (slow) rate $1 / t$ in [7]. 
For the viscous equation

$$
\left\{\begin{array}{l}
u_{t}+f(u)_{x}=\varepsilon u_{x x} \quad(0<x<1, \quad t>0), \\
u(0, x)=u_{0}(x) \quad(0<x<1), \\
u(t, 0)=u(t, 1), \quad u_{x}(t, 0)=u_{x}(t, 1) \quad(t>0),
\end{array}\right.
$$

and the homogeneous equation with positive linear term

$$
\begin{cases}u_{t}+f(u)_{x}+\varepsilon u=0 & (0<x<1, \quad t>0), \\ u(0, x)=u_{0}(x) & (0<x<1), \\ u(t, 0)=u(t, 1) \quad(t>0),\end{cases}
$$

solutions decay at a faster rate assuming $\varepsilon$ is a positive constant. The fast decay for each equation gives a sharp enough estimate to demonstrate the existence of a fixed point of the Poincare map $u(0, x) \mapsto u(T, x)$ for each equation with the time-periodic outer force $g(t, x)$. For systems of conservation laws, Matsumura-Nishida ([9]) proved the existence of periodic solutions for viscous isothermal gas equations for any large periodic outer force, and Matsumura-Yanagi ([10]), Yanagi $([16])$ extended the results to the case of viscous isentropic gases. Feireisl ([6]) proved the existence of periodic solutions for systems of hyperbolic conservation laws with positive linear term. For such systems, solutions of the approximation for the homogeneous systems decay fast uniformly, and he made the sequence of the periodic solutions for the viscous approximation and showed the convergence by using the compensated compactness theory.

However, even in the scalar case, we cannot obtain the time-periodic solution of our problem as the limit of the periodic solutions of equation added $g(t, x)$ to (5) and (6) as $\varepsilon \rightarrow 0$. This is because the estimates for fast decay depend on the constant $\varepsilon$ and are not valid in the limit $\varepsilon \rightarrow 0$, and, according to [7], it does not imply that the decay is fast for the limit equation (4).

On the other hand, the standard method for the proof of the existence of the weak solution to the equation (1) is to show the convergence of some 
approximate solutions which are constructed by a difference scheme method or the artificial viscosity method (5).

Tadmor ([13]) proved the slow decay for the Lax-Friedrichs difference approximations, which does not depend on the mesh size. Note that this is obtained from Oleinik's entropy condition ([11]) and from the periodicity of the boundary condition. We can to solve our problem by such a uniform estimate.

We remark that any such uniform estimates have not been obtained for the approximations of systems of conservation laws for large initial data, and therefore the existence of periodic solutions for systems of equations is still open.

Our result is the following.

THEOREM 1 Under assumptions (14) and (15) for $f$ and $g$, the problem (1), (7) has a time periodic weak solution $u(t, x)$ for any average $\bar{u}$, and the solution satisfies the entropy condition (13).

We remark that the periodic solution of the problem $(1),(7)$ is not unique with respect to $g$ and $\bar{u}$.

The outline of the proof of Theorem 1 is the followings. We construct a Lax-Friedrichs difference approximation for the problem (1), (7) and we will obtain the estimate of uniform bounds for it by the methods similar to those of Tadmor $([13])$ in $\S 4$. The Poincare map is regarded as on the finite dimensional space for the difference approximation because the approximate solution has values at each finite point for fixed $t$. We show the map takes a closed convex set into the same set. Hence we can use Brouwer's fixed point theorem for the existence of the fixed point of the map from the continuity of the approximation. Since these fixed points are uniformly bounded with respect to the any mesh lengths, we obtain a subsequence that converges to a weak solution 
by the compensated compactness theory $([15])$. We check the compactness of the entropy for the approximation in $\S 5$ for the last convergence, and show the limit is a weak solution of the problem (1), (7) satisfying the entropy condition in $\S 6$.

\section{Preliminaries}

A function $u(t, x)$ is called the weak solution of the time-periodic problem (1) and

$$
\begin{cases}u(t, 0)=u(t, 1) & (0<t<T), \\ u(0, x)=u(T, x) & (0<x<1)\end{cases}
$$

with period $T$ if the function $u(t, x)$ is bounded measurable in the region $(0, T) \times(0,1)$, and there exists a bounded measurable function $\bar{u}(x)$ such that space-periodic extensions of $u(t, x), g(t, x)$ and $\bar{u}(x)$ satisfy

$$
\iint_{0<t<T}\left(u \phi_{t}+f(u) \phi_{x}+g \phi\right) d x d t+\int_{R} \bar{u}(x)\{\phi(0, x)-\phi(T, x)\} d x=0(8)
$$

for any $\phi(t, x) \in C_{0}^{1}\left([0, T] \times R_{x}\right)$.

Note that the definition has other equivalent forms. One is the following:

$$
\iint_{[0, T] \times[0,1]}\left(u \phi_{t}+f(u) \phi_{x}+g \phi\right) d x d t+\int_{0}^{1} \bar{u}(x)\{\phi(0, x)-\phi(T, x)\} d x=0(9)
$$

for any $\phi(t, x) \in C_{0}^{1}([0, T] \times[0,1]), \phi(t, 0)=\phi(t, 1) \quad(0<t<T)$. Another is expressed in terms of the space-periodic extension of $\bar{u}$ and for the space and time periodic extension of $u(t, x)$. That is, it is required to satisfy

$$
\iint_{t>0}\left(u \phi_{t}+f(u) \phi_{x}+g \phi\right) d x d t+\int_{R} \bar{u}(x) \phi(0, x) d x=0,
$$

for any $\phi(t, x) \in C_{0}^{1}([0, \infty) \times R)$. The conclusion is that $u(t, x)$ satisfies $(9)$ if and only if the followings valid

1. $u$ is solution of (1) in weak sense

$$
u_{t}+f(u)_{x}=g \quad \text { distribution sense in }(0, T) \times(0,1)
$$


2. $u(t, x)$ converges in weak sense to $u(x)$ as $t$ tends to zero and as $t$ tends to $T$

$$
\frac{1}{\varepsilon} \int_{0}^{\varepsilon} u(t, x) d t, \quad \frac{1}{\varepsilon} \int_{T-\varepsilon}^{T} u(t, x) d t \rightarrow u_{0}(x) \quad \text { as } \quad \varepsilon \downarrow 0
$$

in $L^{\infty}(0,1)$ weak*.

3. $f(u(t, \varepsilon))$ and $f(u(t, 1-\varepsilon))$ converge in weak sense to the same value

$$
\frac{1}{\varepsilon} \int_{0}^{\varepsilon} f(u(t, x)) d x, \quad \frac{1}{\varepsilon} \int_{1-\varepsilon}^{1} f(u(t, x)) d x \rightarrow \bar{f}(t) \quad \text { as } \quad \varepsilon \downarrow 0
$$

in $L^{\infty}(0, T)$ weak* for some function $\bar{f}(t)$ of $L^{\infty}(0, T)$.

It seems that the function $u$ which satisfies above conditions is a solution of the problem (1) satisfying

$$
\begin{cases}f(u(t, 0))=f(u(t, 1)) & (0<t<T), \\ u(0, x)=u(T, x) & (0<x<1)\end{cases}
$$

instead of (7). Certainly, both definitions are equivalent for the weak solution. However, these include different means for the entropy condition. The boundary condition of the original problem (7) seems to say that the space-periodic extension satisfies the equation (1), but the problem (11) does not seem to require it. Hence in the case that boundary values of the weak solution $u(t, 0)$ and $u(t, 1)$ are different, these should satisfy the entropy condition, that is,

$$
f^{\prime}(u(t, 1))>0>f^{\prime}(u(t, 0))
$$

for (7), but should not for (11). It remains an open problem whether the boundary condition (11) is well-posed.

A smooth function pair of $u$

$$
(U(u), F(u))
$$


is an entropy pair for the scalar conservation law

$$
u_{t}+f(u)_{x}=0
$$

if

$$
U(u(t, x))_{t}+F(u(x, t))_{x}=0
$$

for a smooth solution of (12). This is equivalent that $U$ and $F$ satisfy

$$
F^{\prime}(u)=U^{\prime}(u) f^{\prime}(u)
$$

The function $U$ called entropy and $F$ called entropy flux. A weak solution $u$ satisfies the entropy condition if

$$
U(u(t, x))_{t}+F(u(x, t))_{x} \leq U^{\prime}(u(t, x)) g(t, x) \quad \text { in }(0, T) \times R
$$

for any smooth entropy pair with convex entropy $U$.

We suppose that the function $f(u)$ is smooth,

$$
f^{\prime \prime}(u) \geq \delta>0 \quad(u \in R), \quad \int_{0}^{1} d x \int_{0}^{T} g(t, x) d t=0
$$

and $g(t, x)$ is a time-periodic function with period $T$. The last relation for $g(t, x)$ is need for the existence of a time periodic solution.

We also assume that the space-extension of $g(t, x)$ satisfies

$$
g(t, x)-g(t, y) \leq G_{1}(x-y) \quad(x>y)
$$

for any $x, y$ and $t$, where $G_{1}$ is a constant. The condition is necessary by a technical reason.

It is well-known that the solution of the Riemann problem for the scalar conservation law

$$
\left\{\begin{array}{l}
u_{t}+f(u)_{x}=0 \quad(t>0, x \in R), \\
u(0, x)= \begin{cases}u_{l} & (x<0) \\
u_{r} & (x>0)\end{cases}
\end{array}\right.
$$


is each of the two typical waves. In the case $u_{l}<u_{r}$ the solution is the rarefaction wave

$$
u(t, x)= \begin{cases}u_{l} & \left(x / t<f^{\prime}\left(u_{l}\right)\right) \\ \left(f^{\prime}\right)^{-1}(x / t) & \left(f^{\prime}\left(u_{l}\right)<x / t<f^{\prime}\left(u_{r}\right)\right) \\ u_{r} & \left(x / t>f^{\prime}\left(u_{r}\right)\right)\end{cases}
$$

and in the case $u_{l}>u_{r}$ the solution is the shock wave

$$
u(t, x)= \begin{cases}u_{l} & (x / t<s), \\ u_{r} & (x / t>s),\end{cases}
$$

where the shock speed $s$ is determined by the Rankine-Hugoniot relation

$$
f\left(u_{r}\right)-f\left(u_{l}\right)=s\left(u_{r}-u_{l}\right)
$$

and Lax's entropy condition

$$
f^{\prime}\left(u_{l}\right)>s>f^{\prime}\left(u_{r}\right)
$$

(cf. [7], [12]).

We note that the average on the line $t=\Delta t$

$$
\frac{1}{2 \Delta x} \int_{-\Delta x}^{\Delta x} u(\Delta t, x) d x
$$

for the solution of the Riemann problem is equal to the Lax-Friedrichs difference approximation

$$
\frac{u_{r}+u_{l}}{2}-\frac{\Delta t}{2 \Delta x}\left\{f\left(u_{r}\right)-f\left(u_{l}\right)\right\}
$$

provided the Courant-Friedrichs-Lewy (CFL) condition

$$
\frac{\Delta x}{\Delta t}>\max \left\{\left|f^{\prime}\left(u_{l}\right)\right|,\left|f^{\prime}\left(u_{r}\right)\right|\right\} .
$$




\section{Approximate Solution}

In this section, we construct an approximate solution of the Lax-Friedrichs difference scheme type for the initial-boundary value problem (1), (2) in a standard way (cf. [3],[4],[14].)

Let the initial data $u(0, x)$ be a bounded measurable function and

$$
\|u(0, \cdot)\|_{L^{\infty}} \leq M
$$

We suppose that the inverse of the $x$-mesh length $\Delta x$ and the ratio of the period $T$ and the $t$-mesh length are even integers $2 L$ and $2 N$,

$$
2 L \Delta x=1, \quad 2 N \Delta t=T \text {. }
$$

It is necessary that the ratio of $\Delta x$ and $t$-mesh length $\Delta t$ is a sufficiently large constant for the CFL condition. We take the value such that

$$
\frac{\Delta x}{\Delta t} \geq \Lambda \equiv \max _{|u| \leq M+T G_{0}}\left|f^{\prime}(u)\right|
$$

where $T$ is time-period of function $g$, and $G_{0}$ is the maximum value of $|g|$

$$
G_{0}=\max _{[0, T] \times[0,1]}|g(t, x)| .
$$

Let $E_{j}^{n}$ be an interval and $J_{n}$ an index set such that

$$
\left\{\begin{aligned}
E_{j}^{n} & =((j-1) \Delta x,(j+1) \Delta x] \quad\left(j \in J_{n}, n=0,1,2, \ldots\right), \\
J_{n} & = \begin{cases}\{\ldots,-5,-3,-1,1,3,5, \ldots\} & \text { if } n \text { is even } \\
\{\ldots,-4,-2,0,2,4, \ldots\} & \text { if } n \text { is odd }\end{cases}
\end{aligned}\right.
$$

and we denote $R\left(t, x ; u_{l}, u_{r}\right)$ as the solution of the Riemann problem (16).

The approximation of the initial value $u^{\Delta}(0, x)$ is defined as a step value function

$$
u^{\Delta}(0, x)=u_{j}^{0} \equiv \frac{1}{m\left(E_{j}^{0}\right)} \int_{E_{j}^{0}} u(0, x) d x \quad \text { on } E_{j}^{0} \quad\left(j \in J_{0}\right),
$$


where $u(0, x)$ is extended to the function on $R$ as the $x$-periodic function. The value $u_{j}^{0}$ has the periodicity of $u_{j+2 L}^{0}=u_{j}^{0}$. We define $u^{\Delta}(t, x)$ as the solution of the Riemann problem for the step initial data $u^{\Delta}(0, x)$

$$
u^{\Delta}(t, x)=R\left(t, x-j \Delta x, u_{j-1}^{0}, u_{j+1}^{0}\right)
$$

in each small regions $(0, \Delta t) \times E_{j}^{1}, j \in J_{1}$. The wave must arrive at the top of the region by the CFL condition (17). On the line $t=\Delta t$, we define $u^{\Delta}(\Delta t, x)$ as the mean value

$$
u^{\Delta}(\Delta t, x)=u_{j}^{1} \equiv \frac{1}{m\left(E_{j}^{1}\right)} \int_{E_{j}^{1}} u^{\Delta}(\Delta t-0, x) d x \quad \text { on } E_{j}^{1} \quad\left(j \in J_{1}\right),
$$

and construct $u^{\Delta}(t, x)$ by solutions of Riemann problems in $(\Delta t, 2 \Delta t) \times R$ similarly in $(0, \Delta t) \times R$,

$$
u^{\Delta}(t, x)=R\left(t-\Delta t, x-j \Delta x, u_{j-1}^{1}, u_{j+1}^{1}\right) \quad \text { in }(\Delta t, 2 \Delta t) \times E_{j}^{2} \quad\left(j \in J_{2}\right) .
$$

On $t=2 \Delta t$, we set $u^{\Delta}(2 \Delta t, x)$ as the sum of the mean value and the term for the outer force

$$
\begin{aligned}
& u^{\Delta}(2 \Delta t, x)=u_{j}^{2}=u_{0, j}^{2}+2 \Delta t g_{j}^{1} \\
& \equiv \frac{1}{m\left(E_{j}^{2}\right)} \int_{E_{j}^{2}} u^{\Delta}(2 \Delta t-0, x) d x+\frac{1}{m\left(E_{j}^{2}\right)} \int_{0}^{2 \Delta t} d t \int_{E_{j}^{2}} g(t, x) d x \\
& \quad \text { on } E_{j}^{2} \quad\left(j \in J_{2}\right) .
\end{aligned}
$$

The last calculation is called the fractional step method.

For $(2 n \Delta t,(2 n+2) \Delta t] \times R(n=1,2, \ldots, N-1)$ we define the approximation $u^{\Delta}(t, x)$ by the similar way. The following lemma shows that above construction can be continued to $n=N-1$.

LEMMA 2 If constants $u_{l}$ and $u_{r}$ satisfy

$$
\left|u_{l}\right| \leq A, \quad\left|u_{r}\right| \leq A,
$$

then the solution of the Riemann problem (16) $u(t, x)$ satisfies $|u(t, x)| \leq A$. 
By Lemma 2, it is easy to show that $\left|u^{\Delta}(t, x)\right| \leq M+T G_{0}$ and waves appeared in the definition of $u^{\Delta}(t, x)$ cannot access in $(0, T) \times R$ from the CFL condition (17) because speeds of these waves do not over the maximum value of $\left|f^{\prime}(u)\right|$.

\section{Decay estimates}

In this section, we obtain the estimate for the approximation $u^{\Delta}(t, x)$ constructed in the last section by the similar way to Tadmor ([13]) for fractional step Lax-Friedrichs difference approximation.

The step values $u_{j}^{n}=u(n \Delta t, j \Delta x)$ are able to be described as forms of the Lax-Friedrichs difference scheme

$$
\left\{\begin{aligned}
& u_{j}^{2 n+1}= \frac{u_{j+1}^{2 n}+u_{j-1}^{2 n}}{2}-\frac{\Delta t}{2 \Delta x}\left(f_{j+1}^{2 n}-f_{j-1}^{2 n}\right) \quad\left(j \in J_{2 n+1}\right), \\
& u_{j}^{2 n+2}= \frac{u_{j+1}^{2 n+1}+u_{j-1}^{2 n+1}}{2}-\frac{\Delta t}{2 \Delta x}\left(f_{j+1}^{2 n+1}-f_{j-1}^{2 n+1}\right)+2 \Delta t g_{j}^{2 n+1} \\
&\left(j \in J_{2 n+2}\right) \\
&(n=0,1,2, \ldots)
\end{aligned}\right.
$$

where $f_{j}^{n}=f\left(u_{j}^{n}\right)$ and

$$
g_{j}^{n}=\frac{1}{4 \Delta t \Delta x} \int_{(n-1) \Delta t}^{(n+1) \Delta t} d t \int_{(j-1) \Delta x}^{(j+1) \Delta x} g(t, x) d x .
$$

Let $v_{j}^{n}$ be the $x$-backward difference of $u_{j}^{n}$

$$
v_{j}^{n}=\frac{u_{j}^{n}-u_{j-2}^{n}}{2 \Delta x}
$$

Then,

$$
\begin{aligned}
& v_{j}^{2 n+1}=\frac{u_{j}^{2 n+1}-u_{j-2}^{2 n+1}}{2 \Delta x} \\
& =\frac{u_{j+1}^{2 n}-u_{j-3}^{2 n}}{4 \Delta x}-\frac{\Delta t}{4(\Delta x)^{2}}\left(f_{j+1}^{2 n}-2 f_{j-1}^{2 n}+f_{j-3}^{2 n}\right) \\
& =\frac{v_{j+1}^{2 n}+v_{j-1}^{2 n}}{2}-\frac{\Delta t}{2 \Delta x} f^{\prime}\left(u_{j-1}^{2 n}\right)\left(v_{j+1}^{2 n}-v_{j-1}^{2 n}\right)
\end{aligned}
$$




$$
\begin{aligned}
& -\Delta t\left\{\left(v_{j+1}^{2 n}\right)^{2} \int_{0}^{1}(1-\theta) f^{\prime \prime}\left(u_{j-1}^{2 n}+\theta\left(u_{j+1}^{2 n}-u_{j-1}^{2 n}\right)\right) d \theta\right. \\
& \left.+\left(v_{j-1}^{2 n}\right)^{2} \int_{0}^{1}(1-\theta) f^{\prime \prime}\left(u_{j-1}^{2 n}+\theta\left(u_{j-3}^{2 n}-u_{j-1}^{2 n}\right)\right) d \theta\right\} \\
\leq & \frac{v_{j+1}^{2 n}+v_{j-1}^{2 n}}{2}-\frac{\Delta t}{2 \Delta x} f^{\prime}\left(u_{j-1}^{2 n}\right)\left(v_{j+1}^{2 n}-v_{j-1}^{2 n}\right)-\Delta t \delta \frac{\left(v_{j+1}^{2 n}\right)^{2}+\left(v_{j-1}^{2 n}\right)^{2}}{2} .
\end{aligned}
$$

Similarly,

$$
\begin{aligned}
v_{j}^{2 n+2} \leq & \frac{v_{j+1}^{2 n+1}+v_{j-1}^{2 n+1}}{2}-\frac{\Delta t}{2 \Delta x} f^{\prime}\left(u_{j-1}^{2 n+1}\right)\left(v_{j+1}^{2 n+1}-v_{j-1}^{2 n+1}\right) \\
& -\Delta t\left\{\delta \frac{\left(v_{j+1}^{2 n+1}\right)^{2}+\left(v_{j-1}^{2 n+1}\right)^{2}}{2}-2 G_{1}\right\},
\end{aligned}
$$

where $G_{1}$ is the value in (15). Let $N^{n}=\max _{j} v_{j}^{n} . N^{n}$ is non-negative because the summation

$$
\sum_{j \in J_{n}, 0 \leq j<2 L} v_{j}^{n}
$$

equals zero from the space periodicity of $u_{j}^{n}$. A function

$$
h(x)=\frac{A}{2} x-\frac{\Delta t \delta}{2} x^{2} \quad(A>0)
$$

increases for $x \leq A /(2 \Delta t \delta)$. Hence, if

$$
N^{2 n} \leq \frac{1}{2 \Delta t \delta}\left(1-\frac{\Delta t}{\Delta x} \Lambda\right)
$$

then

$$
N^{2 n+1}=\max _{j} v_{j}^{2 n+1} \leq N^{2 n}-\Delta t \delta\left(N^{2 n}\right)^{2} .
$$

Similarly,

$$
N^{2 n+1} \leq \frac{1}{2 \Delta t \delta}\left(1-\frac{\Delta t}{\Delta x} \Lambda\right)
$$

yields

$$
N^{2 n+2}=\max _{j} v_{j}^{2 n+1} \leq N^{2 n}-\Delta t\left\{\delta\left(N^{2 n}\right)^{2}-2 G_{1}\right\} .
$$

The simple estimate for $N^{n}$

$$
N^{n}=\max _{j} v_{j}^{n}=\max _{j} \frac{u_{j}^{n}-u_{j-2}^{n}}{2 \Delta x} \leq \frac{M+T G_{0}}{\Delta x}
$$


shows that if

$$
2 \delta\left(M+T G_{0}\right)+\Lambda \leq \frac{\Delta x}{\Delta t},
$$

then

$$
\frac{N^{2 n+2}-N^{2 n}}{2 \Delta t} \leq G_{1}-\frac{\delta}{2}\left(N^{2 n}\right)^{2}
$$

for $n \leq N-1$ since

$$
\begin{aligned}
N^{2 n+1} & \leq N^{2 n}-\Delta t \delta\left(N^{2 n}\right)^{2} \leq N^{2 n} \\
N^{2 n+2} & \leq N^{2 n+1}-\Delta t \delta\left(N^{2 n+1}\right)^{2}+2 \Delta t G_{1} \\
& \leq N^{2 n}-\Delta t \delta\left(N^{2 n}\right)^{2}+2 \Delta t G_{1} .
\end{aligned}
$$

The solution $y(t)$ of ordinary differential equation

$$
\left\{\begin{array}{l}
y^{\prime}=G_{1}-\frac{\delta}{2} y^{2} \\
y(0)=N^{0}
\end{array}\right.
$$

tends to the value

$$
\alpha=\sqrt{2 G_{1} / \delta}
$$

as $t$ tends to infinity. If $N^{0}>\alpha$ then $y(t)$ is monotone decreasing convex function, and if $N^{0}<\alpha$ then $y(t)$ is monotone increasing concave one. In the latter case, the tangent line of $y(t)$ started $t=t_{0}$ across the line $y=\alpha$ at

$$
t=t_{0}+\frac{2}{\delta\left(y\left(t_{0}\right)+\alpha\right)}
$$

and the time is not smaller than $t_{0}+1 /(\delta \alpha)$. Hence, if $N^{2 n} \leq \alpha$ then $N^{2 m} \leq \alpha$ for $n \leq m \leq N$ provided that

$$
2 \Delta t \leq \frac{1}{\delta \alpha}
$$

from the inequality (20). In the case $N^{0}>\alpha$, it is easy to show that

$$
N^{2 m} \leq y(2 m \Delta t) \leq \alpha+\frac{2 \alpha}{e^{2 m \alpha \delta \Delta t}-1} \quad(m \leq N) .
$$


We consider the estimate for $u_{j}^{2 N}$ using above estimates for $v_{j}^{n}$. For the summation of $u_{j}^{2 N}$

$$
\begin{aligned}
2 \Delta & x \sum_{j=1}^{L} u_{2 j-1}^{2 N} \\
& =2 \Delta x \sum_{j=1}^{L}\left\{\frac{u_{2 j}^{2 N-1}+u_{2 j-2}^{2 N-1}}{2}-\frac{\Delta t}{2 \Delta x}\left(f_{2 j}^{2 N-1}-f_{2 j-2}^{2 N-1}\right)+2 \Delta t g_{2 j-1}^{2 N-1}\right\} \\
& =2 \Delta x \sum_{j=1}^{L} u_{2 j}^{2 N-1}+4 \Delta x \Delta t \sum_{j=1}^{L} g_{2 j-1}^{2 N-1} \\
& =2 \Delta x \sum_{j=1}^{L} u_{2 j-1}^{2 N-2}+4 \Delta x \Delta t \sum_{j=1}^{L} g_{2 j-1}^{2 N-1} \\
& =2 \Delta x \sum_{j=1}^{L} u_{2 j-1}^{0}+4 \Delta x \Delta t \sum_{n=1}^{N} \sum_{j=1}^{L} g_{2 j-1}^{2 n-1} \\
& =\int_{0}^{1} u_{0}(x) d x+\int_{0}^{T} d t \int_{0}^{1} g(t, x) d x \\
& =\bar{u}
\end{aligned}
$$

from (3).

Next simple lemma is used to the estimate for $u_{j}^{2 N}$.

LEMMA 3 Let $p_{j}$ be real values which satisfy

$$
p_{1}+p_{2}+\cdots+p_{K}=0
$$

and let

$$
q_{j}= \begin{cases}p_{j}-p_{j-1} & (j=2,3, \ldots, K), \\ p_{1}-p_{K} & (j=1)\end{cases}
$$

Then

$$
\max _{j}\left|p_{j}\right| \leq \sum_{j=1}^{K}\left|q_{j}\right| \leq 2 K \max _{j} q_{j} .
$$

The proof is omitted.

Let $K=L, p_{j}=u_{2 j-1}^{2 N}-\bar{u}$ in Lemma 3 , then $q_{j}=2 v_{2 j-1}^{2 N} \Delta x$ and

$$
\left|u_{j}^{2 N}-\bar{u}\right| \leq 2 L \max _{j} 2 \Delta x v_{j}^{2 N}=4 L \Delta x N^{2 N} \leq 2 \alpha+\frac{4 \alpha}{e^{\alpha \delta T}-1}
$$

from (23). 
PROPOSITION 4 Under assumptions (19), (22)

$$
\left|u_{j}^{2 N}-\bar{u}\right| \leq 2 \alpha+\frac{4 \alpha}{e^{\alpha \delta T}-1} .
$$

Proposition 4 give the time-periodic solution of the difference approximation.

Let $A$ be sufficiently large number such that

$$
A>2 \alpha+\frac{4 \alpha}{e^{\alpha \delta T}-1}
$$

and let $M=|\bar{u}|+A$. We take mesh lengths $\Delta x$ and $\Delta t$ to satisfy (19), (22) and

$$
\frac{\Delta x}{\Delta t} \leq \Lambda_{2} \equiv 2\left\{2 \delta\left(M+T G_{0}\right)+\Lambda\right\}
$$

for the estimation of the entropy $(\S 5)$. Let $D_{L}$ be a set

$$
\begin{aligned}
D_{L} & =D_{L}(\bar{u}, A) \\
& =\left\{\left(u_{1}, u_{2}, \ldots, u_{L}\right) \in R^{L} ; \quad \sum_{j=1}^{L} u_{j}=\bar{u}, \quad \max \left|u_{j}-\bar{u}\right| \leq A\right\} .
\end{aligned}
$$

If we take $\left(u_{1}^{0}, u_{3}^{0}, \ldots, u_{2 L-1}^{0}\right)$ from $D_{L}$, set $u_{j}^{0}$ periodically for any $j \in J_{0}$, then we can construct the approximation $u^{\Delta}(t, x)$ by the way in $\S 3$. Obviously $D_{L}$ is a closed convex set and the range of the mapping

$$
D_{L} \ni\left(u_{1}^{0}, u_{3}^{0}, \ldots u_{2 L-1}^{0}\right) \mapsto\left(u_{1}^{2 N}, u_{3}^{2 N}, \ldots u_{2 L-1}^{2 N}\right)
$$

is contained in $D_{L}$ from Proposition 4 . Hence, we obtain the fixed point

$$
\left(\bar{u}_{1}^{0}, \bar{u}_{3}^{0}, \ldots, \bar{u}_{2 L-1}^{0}\right)
$$

of the mapping from Brouwer's fixed point theorem in $D_{L}$. Of course, the fixed point depends on $\Delta x$. 


\section{Compactness of entropy}

In this section, we show the compactness of

$$
\left\{U\left(u^{\Delta}(t, x)\right)_{t}+F\left(u^{\Delta}(t, x)\right)_{x} ; \quad\left(u_{1}^{0}, u_{3}^{0}, \ldots, u_{2 L-1}^{0}\right) \in D_{L}, L \geq L_{0}\right\}
$$

in $H_{\text {loc }}^{-1}((0, T) \times(0,1))$ for any smooth entropy pair $(U(u), F(u))$, where $L_{0}$ is sufficiently large integer for (19) and (22).

For simplify, we set $u_{0,+}^{2 n}(x)$ as the function consists of the step values $u_{0, j}^{2 n}$

$$
u_{0,+}^{2 n}(x)=u_{0, j}^{2 n} \quad\left(x \in E_{j}^{2 n}\right)
$$

and use notations

$$
u_{+}^{n}(x)=u(n \Delta t+0, x), \quad u_{-}^{n}(x)=u(n \Delta t-0, x), \quad f_{j}^{n}=f\left(u_{j}^{n}\right),
$$

and so on.

We consider for a particular entropy pairs $\left(U^{*}, F^{*}\right)$ defined by

$$
U^{*}=\frac{u^{2}}{2}, \quad F^{*}=\int_{0}^{u} u f^{\prime}(u)=u f-\int_{0}^{u} f .
$$

Since the approximation $u^{\Delta}(t, x)$ satisfies the equation (12) almost everywhere,

$$
\begin{aligned}
0= & \int_{0}^{T} d t \int_{0}^{1}\left\{U^{*}\left(u^{\Delta}(t, x)\right)_{t}+F^{*}\left(u^{\Delta}(t, x)\right)_{x}\right\} d x \\
= & \sum_{n=1}^{N}\left(\int_{(2 n-2) \Delta t}^{(2 n-1) \Delta t} d t+\int_{(2 n-1) \Delta t}^{2 n \Delta t} d t\right) \int_{0}^{1}\left(U_{t}^{*}+F_{x}^{*}\right) d x \\
= & \sum_{n=1}^{N} \int_{0}^{1}\left(\left[U^{*}\right]_{(2 n-2) \Delta t+0}^{(2 n-1) \Delta t-0}+\left[U^{*}\right]_{(2 n-1) \Delta t+0}^{2 n \Delta t-0}\right) d x \\
& +\int_{0}^{T} \sum_{n=1}\left(\sigma\left[U^{*}\right]-\left[F^{*}\right]\right) d t \\
= & \sum_{n=1}^{N} \int_{0}^{1}\left[U^{*}\right]_{(2 n-1) \Delta t+0}^{(2 n-1) \Delta t-0} d x+\sum_{n=1}^{N} \int_{0}^{1}\left[U^{*}\right]_{2 n \Delta t+0}^{2 n \Delta t-0} d x+\Sigma^{*} \\
& +\int_{0}^{1} U^{*}(T+0, x) d x-\int_{0}^{1} U^{*}(+0, x) d x \\
= & \sum_{n=1}^{N} \sum_{j=0}^{L-1} \int_{E_{2 j}^{2 n-1}}\left[U^{*}\right]_{(2 n-1) \Delta t+0}^{(2 n-1) \Delta t-0} d x+\sum_{n=1}^{N} \sum_{j=1}^{L} \int_{E_{2 j-1}^{2 n}}\left\{\left(U^{*}\right)_{-}^{2 n}-\left(U^{*}\right)_{0,+}^{2 n}\right\} d x \\
& +\Sigma^{*}+I^{*}+J^{*},
\end{aligned}
$$


where

$$
\begin{aligned}
J^{*} & =\sum_{n=1}^{N} \sum_{j=1}^{L} \int_{E_{2 j-1}^{2 n}}\left\{\left(U^{*}\right)_{0,+}^{2 n}-\left(U^{*}\right)_{+}^{2 n}\right\} d x, \\
I^{*} & =\int_{0}^{1} U^{*}(T+0, x) d x-\int_{0}^{1} U^{*}(+0, x) d x, \\
\Sigma^{*} & =\int_{0}^{T} \sum_{\text {shock }}\left(\sigma\left[U^{*}\right]-\left[F^{*}\right]\right) d t,
\end{aligned}
$$

$\sum_{\text {shock }}$ is summation for all the shock waves arise in $[0, T] \times[0,1],\left[U^{*}\right]$ and $\left[F^{*}\right]$ is the difference across the wave, and $\sigma$ is the speed of it. Because of

$$
\begin{aligned}
& \left(U^{*}\right)^{\prime \prime}=1, \quad u_{+}^{2 n-1}=\frac{1}{m\left(E_{2 j}^{2 n-1}\right)} \int_{E_{2 j}^{2 n-1}} u_{-}^{2 n-1} d x \quad\left(x \in E_{2 j}^{2 n-1}\right), \\
& \text { and } \quad u_{0,+}^{2 n}=\frac{1}{m\left(E_{2 j-1}^{2 n}\right)} \int_{E_{2 j-1}^{2 n}} u_{-}^{2 n} d x \quad\left(x \in E_{2 j-1}^{2 n}\right),
\end{aligned}
$$

we have

$$
\frac{1}{2} \sum_{n=1}^{N} \int_{0}^{1}\left|u_{-}^{2 n-1}-u_{+}^{2 n-1}\right|^{2} d x+\frac{1}{2} \sum_{n=1}^{N} \int_{0}^{1}\left|u_{-}^{2 n}-u_{0,+}^{2 n}\right|^{2} d x+\Sigma^{*}=-I^{*}-J^{*} .
$$

The boundedness of $u^{\Delta}(t, x)$

$$
\left|u^{\Delta}(t, x)\right| \leq M+T G_{0}
$$

shows that

$$
\begin{aligned}
\left|J^{*}\right| & \leq \sum_{n=1}^{N} \sum_{j=1}^{L} \int_{E_{2 j-1}^{2 n}}\left|\left(U^{*}\right)_{0,+}^{2 n}-\left(U^{*}\right)_{+}^{2 n}\right| d x \\
& =\sum_{n=1}^{N} \sum_{j=1}^{L} \int_{E_{2 j-1}^{2 n}}\left|\left(u_{0,+}^{2 n}-u_{+}^{2 n}\right) \frac{u_{0,+}^{2 n}+u_{+}^{2 n}}{2}\right| d x \\
& =\sum_{n=1}^{N} \sum_{j=1}^{L} \int_{E_{2 j-1}^{2 n}} 2 \Delta t\left|g_{2 j-1}^{2 n-1} \frac{u_{0,+}^{2 n}+u_{+}^{2 n}}{2}\right| d x \\
& \leq T G_{0}\left(M+T G_{0}\right), \\
\left|I^{*}\right| & \leq \int_{0}^{1}\left|U^{*}(T+0, x)\right| d x+\int_{0}^{1}\left|U^{*}(+0, x)\right| d x \\
& \leq\left(M+T G_{0}\right)^{2} .
\end{aligned}
$$


Hence we obtain the inequality

$$
\begin{aligned}
& \frac{1}{2} \sum_{n=1}^{N} \int_{0}^{1}\left|u_{-}^{2 n-1}-u_{+}^{2 n-1}\right|^{2} d x+\frac{1}{2} \sum_{n=1}^{N} \int_{0}^{1}\left|u_{-}^{2 n}-u_{0,+}^{2 n}\right|^{2} d x \\
& \quad+\int_{0}^{T} \sum_{\text {shock }}\left(\sigma\left[U^{*}\right]-\left[F^{*}\right]\right) d t \leq C\left(M, T, G_{0}\right),
\end{aligned}
$$

where $C\left(M, T, G_{0}\right)$ is a constant depends on $M, T$ and $G_{0}$.

Let $\phi(t, x)$ be a function in $C_{0}^{\infty}((0, T) \times(0,1))$. The integral

$$
\iint_{[0, T] \times[0,1]}\left\{U\left(u^{\Delta}\right) \phi_{t}+F\left(u^{\Delta}\right) \phi_{x}\right\} d t d x
$$

is divided into four parts

$$
\begin{aligned}
& \iint_{[0, T] \times[0,1]}\left\{U\left(u^{\Delta}\right) \phi_{t}+F\left(u^{\Delta}\right) \phi_{x}\right\} d t d x \\
& \quad=\iint_{[0, T] \times[0,1]}\left\{(U \phi)_{t}+(F \phi)_{x}\right\} d t d x \\
& \quad=\Sigma(\phi)+L_{1}(\phi)+L_{2}(\phi)+L_{3}(\phi),
\end{aligned}
$$

where

$$
\begin{aligned}
\Sigma(\phi) & =\int_{0}^{T} \sum_{\text {shock }}(\sigma[U]-[F]) d t, \\
L_{1}(\phi) & =\sum_{n=1}^{N} \sum_{j=1}^{L-1} \phi_{2 j}^{2 n-1} \int_{E_{2 j}^{2 n-1}}\left(U_{-}^{2 n-1}-U_{+}^{2 n-1}\right) d x \\
& +\sum_{n=1}^{N-1} \sum_{j=1}^{L} \phi_{2 j-1}^{2 n} \int_{E_{2 j-1}^{2 n}}\left(U_{-}^{2 n}-U_{0,+}^{2 n}\right) d x, \\
L_{2}(\phi) & =\sum_{n=1}^{N} \int_{0}^{1} \phi\left(U_{0,+}^{2 n}-U_{+}^{2 n}\right) d x, \\
L_{3}(\phi) & =\sum_{n=1}^{N}\left\{\sum_{j=1}^{L-1} \int_{E_{2 j}^{2 n-1}}\left(\phi-\phi_{2 j}^{2 n-1}\right)\left(U_{-}^{2 n-1}-U_{+}^{2 n-1}\right) d x\right. \\
& \left.+\int_{0}^{\Delta x} \phi\left(U_{-}^{2 n-1}-U_{+}^{2 n-1}\right) d x+\int_{1-\Delta x}^{1} \phi\left(U_{-}^{2 n-1}-U_{+}^{2 n-1}\right) d x\right\} \\
& +\sum_{n=1}^{N-1} \sum_{j=1}^{L} \int_{E_{2 j-1}^{2 n}}\left(\phi-\phi_{2 j-1}^{2 n}\right)\left(U_{-}^{2 n}-U_{0,+}^{2 n}\right) d x,
\end{aligned}
$$


and $\phi_{j}^{n}=\phi(n \Delta t, j \Delta x)$. We estimate $L_{j}(\phi)$

$$
\begin{aligned}
\left|L_{1}(\phi)\right| \leq & \|\phi\|_{C^{0}} \max _{|u| \leq M+T G_{0}}\left|U^{\prime \prime}(u)\right| \\
& \times\left(\sum_{n=1}^{N} \int_{0}^{1}\left|u_{-}^{2 n-1}-u_{+}^{2 n-1}\right|^{2} d x+\sum_{n=1}^{N-1} \int_{0}^{1}\left|u_{-}^{2 n}-u_{0,+}^{2 n}\right|^{2} d x\right) \\
\leq & C\left(U^{\prime \prime}, M, T, G_{0}\right)\|\phi\|_{C^{0}}, \\
\left|L_{2}(\phi)\right| \leq & \|\phi\|_{C^{0}} \max _{|u| \leq M+T G_{0}}\left|U^{\prime}(u)\right| \sum_{n=1}^{N} \sum_{j=1}^{L} \int_{E_{2 j-1}^{2 n}}\left|u_{0,+}^{2 n}-u_{+}^{2 n}\right| d x \\
\leq & \|\phi\|_{C^{0}} \max _{|u| \leq M+T G_{0}}\left|U^{\prime}(u)\right| \sum_{n=1}^{N} \sum_{j=1}^{L} 4 \Delta t \Delta x\left|g_{2 j-1}^{2 n-1}\right| \\
\leq & C\left(U^{\prime}, M, T, G_{0}\right)\|\phi\|_{C^{0}}, \\
\left|L_{3}(\phi)\right| \leq & \|\phi\|_{C^{\beta}}(\Delta x)^{\beta} \\
& \times\left(\sum_{n=1}^{N} \int_{0}^{1}\left|U_{-}^{2 n-1}-U_{+}^{2 n-1}\right| d x+\sum_{n=1}^{N-1} \int_{0}^{1}\left|U_{-}^{2 n}-U_{0,+}^{2 n}\right| d x\right) \\
\leq & \|\phi\|_{C^{\beta}}(\Delta x)^{\beta} \max _{|u| \leq M+T G_{0}}\left|U^{\prime}(u)\right| \sqrt{2 N-1} \\
& \times\left(\sum_{n=1}^{N} \int_{0}^{1}\left|u_{-}^{2 n-1}-u_{+}^{2 n-1}\right|^{2} d x+\sum_{n=1}^{N-1} \int_{0}^{1}\left|u_{-}^{2 n}-u_{0,+}^{2 n}\right|^{2} d x\right)^{1 / 2} \\
\leq & C\left(U^{\prime}, M, T, G_{0}\right) \sqrt{T \Delta x / \Delta t}\|\phi\|_{C^{\beta}}(\Delta x)^{\beta-1 / 2} \\
\leq & C\left(U^{\prime}, M, T, G_{0}, \Lambda_{2}\right)\|\phi\|_{C^{\beta}}(\Delta x)^{\beta-1 / 2}
\end{aligned}
$$

and $\Sigma(\phi)$

$$
|\Sigma(\phi)| \leq\|\phi\|_{C^{0}} \max _{|u| \leq M+T G_{0}}\left|U^{\prime \prime}(u)\right| \Sigma^{*} \leq C\left(U^{\prime \prime}, M, T, G_{0}\right)\|\phi\|_{C^{0}}
$$

by the similar way in [3] (I). Hence the integral (28) is the sum of two linear operators $T_{1}(\phi)$ and $T_{2}(\phi)$ which satisfy

$$
\left|T_{1}(\phi)\right| \leq C\|\phi\|_{C^{0}}, \quad\left|T_{2}(\phi)\right| \leq C\|\phi\|_{C^{\beta}}(\Delta x)^{\beta-1 / 2},
$$

where the constant $C$ depends on $M, T, G_{0}$, maximum value of $U^{\prime}$ and $U^{\prime \prime}$, and $\Lambda_{2}$. This shows the following proposition by the argument in [3] (I).

PROPOSITION 5 For any smooth entropy pair $(U, F)$, the set

$$
\left\{U\left(u^{\Delta}(t, x)\right)_{t}+F\left(u^{\Delta}(t, x)\right)_{x} ; \quad\left(u_{1}^{0}, u_{3}^{0}, \ldots, u_{2 L-1}^{0}\right) \in D_{L}, L \geq L_{0}\right\}
$$


is relatively compact in $H_{\mathrm{loc}}^{-1}((0, T) \times(0,1))$.

Proposition 5 and Tartar's theorem ([15]) yields the existence of subsequence which converges almost everywhere. It also valid for the initial data $\left(\bar{u}_{1}^{0}, \bar{u}_{3}^{0}, \ldots, \bar{u}_{2 L-1}^{0}\right)(\S 4)$.

\section{Convergence to an entropy solution}

In the last section, we saw the existence of a convergent sequence of approximate. In this section, we show that the limit is a weak solution and satisfies the entropy condition in this section.

To prove the limit is a weak solution, it is sufficient that the integral

$$
\begin{aligned}
I= & \iint_{[0, T] \times[0,1]}\left(u^{\Delta} \phi_{t}+f^{\Delta} \phi_{x}+g \phi\right) d t d x+\int_{0}^{1} u^{\Delta}(+0, x) \phi(0, x) d x \\
& -\int_{0}^{1} u^{\Delta}(T+0, x) \phi(T, x) d x
\end{aligned}
$$

tends to zero as $\Delta x$ tends to zero for any $\phi \in C_{0}^{1}([0, T] \times[0,1])$ such that $\phi(t, 0)=\phi(t, 1) \quad(0<t<T)$, because the data $\left(\bar{u}_{1}^{0}, \bar{u}_{3}^{0}, \ldots, \bar{u}_{2 L-1}^{0}\right)$ is uniformly bounded and have a subsequence which converges to a function $\bar{u}(x)$ of $L^{\infty}(0,1)$.

By the similar calculation in $\S 5$, it follows

$$
\begin{aligned}
I= & \iint_{[0, T] \times[0,1]} g \phi d t d x+\sum_{n=1}^{N}\left[\phi u^{\Delta}\right]_{(2 n-1) \Delta t+0}^{(2 n-1) \Delta t-0} d x+\sum_{n=1}^{N}\left[\phi u^{\Delta}\right]_{2 n \Delta t+0}^{2 n \Delta t-0} d x \\
= & \sum_{n=1}^{N}\left(\sum_{j=1}^{L-1} \phi_{2 j}^{2 n-1} \int_{E_{2 j}^{2 n-1}}\left[u^{\Delta}\right] d x+\phi_{0}^{2 n-1} \int_{0}^{\Delta x}\left[u^{\Delta}\right] d x\right. \\
& \left.+\phi_{L}^{2 n-1} \int_{1-\Delta x}^{1}\left[u^{\Delta}\right] d x\right)+\sum_{n=1}^{N} \sum_{j=1}^{L} \phi_{2 j-1}^{2 n} \int_{E_{2 j-1}^{2 n}}\left(u_{-}^{2 n}-u_{0,+}^{2 n}\right) d x \\
& +\sum_{n=1}^{N}\left\{\sum_{j=1}^{L-1} \int_{E_{2 j}^{2 n-1}}\left(\phi-\phi_{2 j}^{2 n-1}\right)\left[u^{\Delta}\right] d x+\int_{0}^{\Delta x}\left(\phi-\phi_{0}^{2 n-1}\right)\left[u^{\Delta}\right] d x\right. \\
& \left.+\int_{1-\Delta x}^{1}\left(\phi-\phi_{L}^{2 n-1}\right)\left[u^{\Delta}\right] d x\right\}
\end{aligned}
$$




$$
\begin{aligned}
& +\sum_{n=1}^{N} \sum_{j=1}^{L} \int_{E_{2 j-1}^{2 n}}\left(\phi-\phi_{2 j-1}^{2 n}\right)\left(u_{-}^{2 n}-u_{0,+}^{2 n}\right) d x \\
& +\iint_{[0, T] \times[0,1]} g \phi d t d x+\sum_{n=1}^{N} \int_{0}^{1} \phi\left(u_{0,+}^{2 n}-u_{+}^{2 n}\right) d x \\
& =I_{1}+I_{2}
\end{aligned}
$$

since $\sigma\left[u^{\Delta}\right]-\left[f^{\Delta}\right]=0$ for shocks from the Rankine-Hugoniot relation and $\phi_{0}^{2 n-1}=\phi_{L}^{2 n-1}$, where

$$
\begin{aligned}
I_{1}= & \sum_{n=1}^{N}\left\{\sum_{j=1}^{L-1} \int_{E_{2 j}^{2 n-1}}\left(\phi-\phi_{2 j}^{2 n-1}\right)\left[u^{\Delta}\right] d x+\int_{0}^{\Delta x}\left(\phi-\phi_{0}^{2 n-1}\right)\left[u^{\Delta}\right] d x\right. \\
& \left.+\int_{1-\Delta x}^{1}\left(\phi-\phi_{L}^{2 n-1}\right)\left[u^{\Delta}\right] d x\right\} \\
& +\sum_{n=1}^{N} \sum_{j=1}^{L} \int_{E_{2 j-1}^{2 n}}\left(\phi-\phi_{2 j-1}^{2 n}\right)\left(u_{-}^{2 n}-u_{0,+}^{2 n}\right) d x, \\
I_{2}= & \iint_{[0, T] \times[0,1]} g \phi d t d x+\sum_{n=1}^{N} \int_{0}^{1} \phi\left(u_{0,+}^{2 n}-u_{+}^{2 n}\right) d x .
\end{aligned}
$$

$I_{1}$ tends to zero because

$$
\begin{aligned}
\left|I_{1}\right| \leq & \|\phi\|_{C^{1}} \Delta x\left(\sum_{n=1}^{N} \int_{0}^{1}\left|u_{-}^{2 n-1}-u_{+}^{2 n-1}\right| d x+\sum_{n=1}^{N} \int_{0}^{1}\left|u_{-}^{2 n}-u_{0,+}^{2 n}\right| d x\right) \\
\leq & \|\phi\|_{C^{1}} \Delta x \sqrt{2 N} \\
& \times\left(\sum_{n=1}^{N} \int_{0}^{1}\left|u_{-}^{2 n-1}-u_{+}^{2 n-1}\right|^{2} d x+\sum_{n=1}^{N} \int_{0}^{1}\left|u_{-}^{2 n}-u_{0,+}^{2 n}\right|^{2} d x\right)^{1 / 2} \\
\leq & C\left(\|\phi\|_{C^{1}}, M, T, G_{0}, \Lambda_{2}\right) \sqrt{\Delta x}
\end{aligned}
$$

For $I_{2}$, we obtain

$$
\begin{aligned}
I_{2} & =\iint_{[0, T] \times[0,1]} g \phi d t d x-\sum_{n=1}^{N} \sum_{j=1}^{L} \int_{E_{2 j-1}^{2 n}} 2 \Delta t g_{2 j-1}^{2 n-1} \phi d x \\
& =\sum_{n=1}^{N} \sum_{j=1}^{L} \int_{(2 n-2) \Delta t}^{2 n \Delta t} d t \int_{E_{2 j-1}^{2 n}} g(t, x)\left\{\phi(t, x)-\tilde{\phi}_{2 j-1}^{2 n}\right\} d x,
\end{aligned}
$$

where

$$
\tilde{\phi}_{j}^{n}=\frac{1}{m\left(E_{j}^{n}\right)} \int_{E_{j}^{n}} \phi(n \Delta t, x) d x
$$


The function $\phi-\tilde{\phi}_{2 j-1}^{2 n}$ tends to zero because

$$
\begin{aligned}
\left|\phi-\tilde{\phi}_{2 j-1}^{2 n}\right| & \leq \frac{1}{2 \Delta x} \int_{E_{2 j-1}^{2 n}}|\phi(t, x)-\phi(2 n \Delta t, y)| d y \leq\|\phi\|_{C^{1}}(2 \Delta x+\Delta t) \\
& \leq\|\phi\|_{C^{1}} \Delta x(2+1 / \Lambda) .
\end{aligned}
$$

Therefore we obtain

$$
|I| \leq C\left(\|\phi\|_{C^{1}}, M, T, G_{0}, \Lambda_{2}\right) \sqrt{\Delta t}
$$

Lastly, we show that the limit satisfies the entropy condition (13). Let $(U, F)$ be a smooth entropy pair and $U$ be a convex. The weak form of the entropy condition is the inequality

$$
\iint_{t>0}\left(U \phi_{t}+F \phi_{x}+U^{\prime} g \phi\right) d t d x \geq 0
$$

for any $\phi \in C^{1}((0, \infty) \times R)$. It is sufficient for the proof to show that

$$
\bar{I}=\int_{0}^{T} d t \int_{0}^{1}\left(U\left(u^{\Delta}\right) \psi_{t}+F\left(u^{\Delta}\right) \psi_{x}+U^{\prime}\left(u^{\Delta}\right) g \psi_{x}\right) d x \geq o(1)
$$

for $\psi \in C^{1}([0, T] \times[0,1])$ such that

$$
\psi \geq 0, \quad \psi(0, x)=\psi(T, x) \quad(x \in[0,1]), \quad \psi(t, 0)=\psi(t, 1) \quad(t \in[0, T])
$$

For convex entropy $U$ we can see

$$
\sigma[U]-[F] \geq 0
$$

(cf. [3]). Hence,

$$
\begin{aligned}
\bar{I}= & \sum_{n=1}^{N} \int_{0}^{1}[U \psi]_{(2 n-1) \Delta t+0}^{(2 n-1) \Delta t-0} d x+\sum_{n=1}^{N} \int_{0}^{1}[U \psi]_{2 n \Delta t+0}^{2 n \Delta t-0} d x+\iint U^{\prime} g \psi d t d x \\
& +\int_{0}^{1}(U \psi)(T+0, x) d x-\int_{0}^{1}(U \psi)(+0, x) d x \\
& +\int_{0}^{T} \sum_{\text {shock }} \phi(\sigma[U]-[F]) d t+\iint U^{\prime} g \psi d t d x \\
\geq & \bar{L}_{1}+\bar{L}_{2}+\bar{L}_{3}+\iint U^{\prime} g \psi d t d x
\end{aligned}
$$


since $u^{\Delta}(T+0, x)=u^{\Delta}(+0, x)$, where

$$
\begin{aligned}
\bar{L}_{1}= & \sum_{n=1}^{N}\left\{\sum_{n=1}^{L} \psi_{2 j}^{2 n-1} \int_{E_{2 j}^{2 n-1}}[U] d x+\psi_{0}^{2 n-1} \int_{0}^{\Delta x}[U] d x\right. \\
& \left.+\psi_{2 L}^{2 n-1} \int_{1-\Delta x}^{1}[U] d x\right\}+\sum_{n=1}^{N} \sum_{j=1}^{L} \psi_{2 j-1}^{2 n} \int_{E_{2 j-1}^{2 n}}\left(U_{-}^{2 n}-U_{0,+}^{2 n}\right) d x, \\
\bar{L}_{2}= & \sum_{n=1}^{N} \int_{0}^{1} \psi\left(U_{0,+}^{2 n}-U_{+}^{2 n}\right) d x, \\
\bar{L}_{3}= & \sum_{n=1}^{N}\left\{\sum_{n=1}^{L} \int_{E_{2 j}^{2 n-1}}\left(\psi-\psi_{2 j}^{2 n-1}\right)[U] d x+\int_{0}^{\Delta x}\left(\psi-\psi_{0}^{2 n-1}\right)[U] d x\right. \\
& \left.+\int_{1-\Delta x}^{1}\left(\psi-\psi_{2 L}^{2 n-1}\right)[U] d x\right\} \\
& +\sum_{n=1}^{N} \sum_{j=1}^{L} \int_{E_{2 j-1}^{2 n}}\left(\psi-\psi_{2 j-1}^{2 n}\right)\left(U_{-}^{2 n}-U_{0,+}^{2 n}\right) d x .
\end{aligned}
$$

By $U^{\prime \prime} \geq 0$ and $\psi_{0}^{2 n-1}=\psi_{2 L}^{2 n-1}, \bar{L}_{1} \geq 0$. It follows by the similar way in $\S 5$ that

$$
\begin{aligned}
\left|\bar{L}_{3}\right| \leq & \|\psi\|_{C^{1}} \Delta x \max \left|U^{\prime}\right| \\
& \times\left(\sum_{n=1}^{N} \int_{0}^{1}\left|u_{-}^{2 n-1}-u_{+}^{2 n-1}\right| d x+\sum_{n=1}^{N} \int_{0}^{1}\left|u_{-}^{2 n}-u_{0,+}^{2 n}\right| d x\right) \\
\leq & C\left(\|\psi\|_{C^{1}}, U^{\prime}, M, T, G_{0}, \Lambda_{2}\right) \sqrt{\Delta x} .
\end{aligned}
$$

Hence we have

$$
\bar{I} \geq \iint_{[0, T] \times[0,1]} U^{\prime} g \psi d t d x+\bar{L}_{2}+O(\sqrt{\Delta x}) .
$$

From Taylor's expansion theorem

$$
\begin{aligned}
\bar{L}_{2}= & \sum_{n=1}^{N} \int_{0}^{1} \psi(2 n \Delta t, x)\left\{U^{\prime}\left(u_{+}^{2 n}\right)\left(u_{0,+}^{2 n}-u_{+}^{2 n}\right)\right\} d x \\
& +\sum_{n=1}^{N} \int_{0}^{1} \psi(2 n \Delta t, x)\left(u_{0,+}^{2 n}-u_{+}^{2 n}\right)^{2} d x \\
& \times \int_{0}^{1}(1-\theta) U^{\prime \prime}\left(u_{+}^{2 n}+\theta\left(u_{0,+}^{2 n}-u_{+}^{2 n}\right)\right) d \theta \\
\geq & -\sum_{n=1}^{N} \sum_{j=1}^{L} 2 \Delta t g_{2 j-1}^{2 n-1} U^{\prime}\left(u_{2 j-1}^{2 n}\right) \int_{E_{2 j-1}^{2 n}} \psi(2 n \Delta t, x) d x .
\end{aligned}
$$


First two terms of the right hand side of (29) become

$$
\begin{aligned}
& \iint_{[0, T] \times[0,1]} U^{\prime} g \psi d t d x+\bar{L}_{2} \\
= & \sum_{n=1}^{N} \sum_{j=1}^{L} \int_{(2 n-2) \Delta t}^{2 n \Delta t} d t \int_{E_{2 j-1}^{2 n}}\left(U^{\prime} \psi\right)_{2 j-1}^{2 n} g d x \\
& +\sum_{n=1}^{N} \sum_{j=1}^{L} \int_{(2 n-2) \Delta t}^{2 n \Delta t} d t \int_{E_{2 j-1}^{2 n}} U^{\prime}\left(\psi-\psi_{2 j-1}^{2 n}\right) g d x \\
& +\sum_{n=1}^{N} \sum_{j=1}^{L} \int_{(2 n-2) \Delta t}^{2 n \Delta t} d t \int_{E_{2 j-1}^{2 n}}+\left\{U^{\prime}-U^{\prime}\left(u_{2 j-1}^{2 n}\right)\right\} \psi_{2 j-1}^{2 n} g d x+\bar{L}_{2} \\
\geq & \sum_{n=1}^{N} \sum_{j=1}^{L} 4 \Delta t \Delta x\left(U^{\prime} \psi\right)_{2 j-1}^{2 n} g_{2 j-1}^{2 n-1} \\
& +\sum_{n=1}^{N} \sum_{j=1}^{L} \psi_{2 j-1}^{2 n} \int_{(2 n-2) \Delta t}^{2 n \Delta t} d t \int_{E_{2 j-1}^{2 n}}\left\{U^{\prime}-U^{\prime}\left(u_{2 j-1}^{2 n}\right)\right\} g d x \\
& -\sum_{n=1}^{N} \sum_{j=1}^{L} 2 \Delta t g_{2 j-1}^{2 n-1} U^{\prime}\left(u_{2 j-1}^{2 n}\right) \int_{E_{2 j-1}^{2 n}} \psi(2 n \Delta t, x) d x+O(\Delta x) \\
\geq & \sum_{n=1}^{N} \sum_{j=1}^{L} \psi_{2 j-1}^{2 n} \int_{(2 n-2) \Delta t}^{2 n \Delta t} d t \int_{E_{2 j-1}^{2 n}}\left\{U^{\prime}-U^{\prime}\left(u_{2 j-1}^{2 n}\right)\right\} g d x+O(\Delta x) \\
= & \sum_{n=1}^{N} \int_{(2 n-2) \Delta t}^{2 n \Delta t} d t \int_{0}^{1}\left\{U^{\prime}-U^{\prime}\left(u_{+}^{2 n}\right)\right\} \psi_{+}^{2 n} g d x+O(\Delta x) \\
= & \sum_{n=1}^{N} \int_{(2 n-2) \Delta t}^{2 n \Delta t} d t \int_{0}^{1}\left\{U^{\prime}-U^{\prime}\left(u_{0,+}^{2 n}\right)\right\} \psi_{+}^{2 n} g d x+O(\Delta x),
\end{aligned}
$$

where $\psi_{+}^{2 n}$ is a step value function defined by

$$
\psi_{+}^{2 n}(x)=\psi_{2 j-1}^{2 n} \quad\left(x \in E_{2 j-1}^{2 n}\right) .
$$

Remember the following lemma proved in [8].

LEMMA 6 Let $u(t, x)$ be the solution of the Riemann problem (16), $\bar{u}$ be the average of $u(\Delta t, x)$

$$
u(t, x)=R\left(t, x ; u_{l}, u_{r}\right), \quad \bar{u}=\frac{1}{2 \Delta x} \int_{-\Delta x}^{\Delta x} u(\Delta t, x) d x
$$

and the maximum wave speed $\Lambda=\max \left\{\left|f^{\prime}\left(u_{l}\right)\right|,\left|f^{\prime}\left(u_{r}\right)\right|\right\}$ satisfy $\Lambda<\Delta x / \Delta t$. Then

$$
\int_{-\Delta x}^{\Delta x}|\bar{u}-u(t, x)| d x \leq C \int_{-\Delta x}^{\Delta x}|\bar{u}-u(\Delta t, x)| d x \quad(0 \leq t \leq \Delta t)
$$


where $C$ is a positive constant

$$
C=(1-\Lambda \Delta t / \Delta x)^{-1}
$$

For the approximation $u^{\Delta}(t, x)$ the constant $C$ is

$$
\left(1-\frac{\Lambda}{2 \delta\left(M+T G_{0}\right)+\Lambda}\right)^{-1}=1+\frac{\Lambda}{2 \delta\left(M+T G_{0}\right)}
$$

from (19). By Lemma 6 we estimate the rest term

$$
\begin{aligned}
& \left|\sum_{n=1}^{N} \int_{(2 n-2) \Delta t}^{2 n \Delta t} d t \int_{0}^{1}\left\{U^{\prime}-U^{\prime}\left(u_{0,+}^{2 n}\right)\right\} \psi_{+}^{2 n} g d x\right| \\
\leq & \sum_{n=1}^{N} \int_{(2 n-1) \Delta t}^{2 n \Delta t} d t \int_{0}^{1}\left|U^{\prime}-U^{\prime}\left(u_{0,+}^{2 n}\right)\right|\left|\psi_{+}^{2 n} g\right| d x \\
& +\sum_{n=1}^{N} \int_{(2 n-2) \Delta t}^{(2 n-1) \Delta t} d t \int_{0}^{1}\left|U^{\prime}-U^{\prime}\left(u_{+}^{2 n-1}\right)\right|\left|\psi_{+}^{2 n} g\right| d x \\
& +\sum_{n=1}^{N} \int_{(2 n-2) \Delta t}^{(2 n-1) \Delta t} d t \int_{0}^{1}\left|U^{\prime}\left(u_{+}^{2 n-1}\right)-U^{\prime}\left(u_{0,+}^{2 n}\right)\right|\left|\psi_{+}^{2 n} g\right| d x \\
\leq & C\left(M, \delta, M, T, G_{0},\|\psi\|_{C^{0}}, \max \left|U^{\prime \prime}\right|\right) \Delta t \\
& \times \sum_{n=1}^{N} \int_{0}^{1}\left(\left|u_{-}^{2 n}-u_{0,+}^{2 n}\right|+\left|u_{-}^{2 n-1}-u_{+}^{2 n-1}\right|+\left|u_{-}^{2 n}-u_{0,+}^{2 n}\right|\right) d x \\
\leq & C \sqrt{\Delta x} .
\end{aligned}
$$

Therefore, we obtain $\bar{I} \geq-C \sqrt{\Delta x}$.

\section{References}

[1] Chen Guiqiang, Convergence of the Lax-Friedrichs scheme for isentropic gas dynamics(III), Acta Math. Sci. 6 (1986), 75-120 (Chinese edition: 8(1988), 101-134).

[2] Chueh K. N., Conley C. C. \& Smoller J. A., Positively invariant regions for systems of nonlinear diffusion equations, Indiana Univ. Math. J. 26 (1977), 373-392. 
[3] Ding Xiaxi, Chen Guiqiang \& Luo Peizhu, Convergence of the LaxFriedrichs scheme for isentropic gas dynamics (I)-(II), Acta Math. Sci. 5 (1985), 415-432, 433-472 (Chinese edition: 7(1987), 467-481; 8(1988), 61-94).

[4] Ding Xiaxi, Chen Guiqiang \& Luo Peizhu, Convergence of the fractional step Lax-Friedrichs scheme and Godunov scheme for the isentropic system of gas dynamics, Comm. Math. Phys. 121 (1989), 63-84.

[5] DiPerna R. J., Convergence of approximate solutions to conservation laws, Arch. Rat. Mech. Anal. 82 (1983), 27-70.

[6] Feireisl E., Time-periodic solutions to quasilinear telegraph equations with large data, Arch. Rat. Mech. Anal. 112 (1990), 45-62.

[7] Lax P. D., Hyperbolic conservation laws II, Comm. Pure Appl. Math. 10 (1957), 537-566.

[8] Makino T. \& Takeno S., Initial boundary value problem for the spherically symmetric motion of isentropic gas, Japan J. Indust. Appl. Math 11 (1994), 171-183.

[9] Matsumura A. \& Nishida T., Periodic solutions of a viscous gas equation, Lecture Notes in Num. Appl. Anal. 10 (1988), 49-82.

[10] Matsumura A. \& Yanagi S., Uniform boundedness of the solutions for a one dimensional isentropic model system of compressible viscous gas, Comm. Math. Phys. 175 (1996), 259-274.

[11] Oleinik O. A., Discontinuous solutions of non-linear differential equations, Usp. Mat. Nauk 12 (1957), 3-73.

[12] Smoller J., Shock waves and reaction-diffusion equations. Springer, New York (1982). 
[13] Tadmor E., The large-time behavior of the scalar, genuinely nonlinear Lax-Friedrichs scheme, Math.Comp. 43 (1984), 353-368.

[14] Takeno S., Initial boundary value problems for isentropic gas dynamics, Proc. Royal Soc. Edinburgh 120A (1992), 1-23.

[15] Tartar L., Compensated compactness and applications to partial differential equations, in Nonlinear analysis and mechanics: Heriot-Watt Symposium, Vol.4, R.J.Knops, ed., Research Notes in Mathematics 39, Pitman, London, (1979), 136-211.

[16] Yanagi S., Global existence and asymptotic behavior of the solutions for one-dimensional compressible fluids.. Ph.D Thesis, Waseda University (1995). 